\title{
Downregulated microRNA-510-5p acts as a tumor suppressor in renal cell carcinoma
}

\author{
DUQUN CHEN ${ }^{1-3}$, YUCHI LI ${ }^{1,2,4}$, ZUHU YU $^{1-3}$, YIFAN LI $^{1-3}$, ZHENGMING SU $^{1,2,4}$, \\ LIANGCHAO NI $^{1,2}$, SHANGQI YANG ${ }^{1,2}$, YAOTING GUI ${ }^{2}$ and YONGQING LAI ${ }^{1,2}$ \\ ${ }^{1}$ Department of Urology, Peking University Shenzhen Hospital, Shenzhen, Guangdong 518036; \\ ${ }^{2}$ The Guangdong and Shenzhen Key Laboratory of Male Reproductive Medicine and Genetics, \\ Institute of Urology of Shenzhen PKU-HKUST Medical Center, Shenzhen, Guangdong 518036; ${ }^{3}$ Department of Urology, \\ Anhui Medical University First Affiliated Hopsital, Hefei, Anhui 230032; ${ }^{4}$ Department of Urology, \\ Shantou University Medical College, Shantou, Guangdong 515041, P.R. China
}

Received June 29, 2014; Accepted March 18, 2015

DOI: $10.3892 / \mathrm{mmr} .2015 .3704$

\begin{abstract}
MicroRNA (miR)-510-5p has been demonstrated to be involved in a number of types of malignancy; however, the function of miR-510-5p in renal cancer remains unclear. The present study aimed to determine the expression of miR-510-5p in renal cell carcinoma (RCC) specimens and analyzed the impact of miR-510-5p on renal cancer by 3-(4,5-dimethylthiazol-2-yl)-2,5-diphenyltetrazolium bromide, wound scratch and apoptosis assays. The results showed that miR-510-5p was significantly downregulated in RCC specimens compared with normal renal specimens. Overexpression of miR-510-5p by synthetic mature mimics reduced cell proliferation and migration and induced an increase in cell apoptosis, indicating that miR-510-5p may act as a tumor suppressor in RCC. The present study firstly revealed that downregulated miR-510-5p functioned as a tumor suppressor by reducing cellular proliferation and migration, and inducing apoptosis in RCC. Further research is required to define target genes of miR-510-5p to determine the cellular mechanism of miR-510-5p in the carcinogenesis of RCC.
\end{abstract}

\section{Introduction}

Renal cell carcinoma (RCC) is the most common neoplasm of the adult kidney with the highest rate of recurrence and mortality

Correspondence to: Professor Yongqing Lai, Department of Urology, Peking University Shenzhen Hospital, 1120 Lianhua Road, Shenzhen, Guangdong 518036, P.R. China

E-mail: yqlord@163.com

Professor Yaoting Gui, The Guangdong and Shenzhen Key Laboratory of Male Reproductive Medicine and Genetics, Institute of Urology of Shenzhen PKU-HKUST Medical Center, 1120 Lianhua Road, Shenzhen, Guangdong 518036, P.R. China

E-mail: gyt@pkuszh.com

Key words: microRNA-510, renal cell carcinoma, tumor suppressor among malignances in urologic systems (1). Nearly $30 \%$ of RCC patients present at advanced stages, and $\sim 40 \%$ of patients that undergo surgical resection experience recurrence during subsequent follow-up $(2,3)$. Metastatic RCC has an extremely poor prognosis and remains an incurable disease despite the great improvement in surgery and personalized treatments $(4,5)$. The high rates of recurrence and mortality of RCC create an urgent requirement for personalized care and reliable biomarkers for early detection and prognosis prediction (6). Therefore, exploring the molecular mechanisms underlying the disease and identifying novel molecular biomarkers is important (7).

In recent years, miRNAs have emerged as important molecules in the complex networks of gene regulation (8). These small, endogenous non-coding RNA molecules that regulate the expression of protein coding genes at a post-transcriptional level have been implicated in a variety of human disorders, such as infectious diseases, metabolic diseases and cancer $(9,10)$. Aberrantly expressed miRNAs are prevalent in a number of types of human cancer, and are important in cancer initiation, development and metastasis $(11,12)$. Certain highly expressed miRNAs may function as oncogenes by repressing tumor suppressors, whereas downregulated miRNAs may function as tumor suppressors by negatively regulating oncogenes (13). Their stable expression in the blood render them reliable biomarkers for early detection, diagnosis and prognosis prediction in various diseases, including cancer $(14,15)$.

miR-510 has been demonstrated to be involved in lung cancer, breast cancer, gastric cancer and ovarian serous carcinoma (16-19); however, the expression and function of miR-510-5p in renal cancer remains unclear. The present study aimed to determine the expression of miR-510-5p in RCC tissues and paired normal adjacent tissues, and analyzed the impact of miR-510-5p on renal cancer by 3-(4,5-dimethylthiazol-2-yl)-2,5-diphenyltetrazolium bromide (MTT), wound scratch and apoptosis assays.

\section{Materials and methods}

Clinical specimens. The present study was approved by the Ethics Committee of Peking University Shenzhen Hospital 
(Shenzhen, China) and Anhui Medical University First Affiliated Hospital (Hefei, China). Written informed consent was obtained from every patient prior to sample collection. A total of 48 paired renal cell carcinoma (RCC) and adjacent normal specimens were collected from patients receiving radical nephrectomies at Peking University Shenzhen Hospital or Anhui Medical University First Affiliated Hospital. All samples were processed and stored at $-80^{\circ} \mathrm{C}$ in RNAlater (Qiagen, Valencia, CA, USA) until RNA isolation. The clinical and pathological information of all the patients is summarized in Table I. These samples were staged according to the American Joint Committee on Cancer (AJCC) staging system (20).

Cell culture and RNA extraction. Two human RCC cell lines, ACHN and 786-O (Guangdong and Shenzhen Key Laboratory of Male Reproductive Medicine and Genetics, Shenzhen, China) were used in this study. They were incubated in Dulbecco's modified Eagle's medium (Invitrogen Life Technologies, Carlsbad, CA, USA) supplemented with $10 \%$ fetal bovine serum (Invitrogen Life Technologies) and maintained in a humidified incubator containing $5 \% \mathrm{CO}_{2}$ at $37^{\circ} \mathrm{C}$. Total-RNA of each sample was isolated with TRIzol reagent (Invitrogen Life Technologies, Carlsbad, CA, USA) and purified with an RNeasy Maxi kit (Qiagen) according to the manufacturer's instructions.

Reverse transcription-quantitative polymerase chain reaction $(R T-q P C R)$. To obtain the cDNA templates, $1 \mu \mathrm{g}$ total RNA of each sample was isolated for reverse transcription with the miScript Reverse Transcription reagent (Qiagen).

PCR amplification was performed using $1 \mu \mathrm{l}$ cDNA in a $20 \mu \mathrm{l}$ reaction system, containing $10 \mu \mathrm{l}$ QuantiTect SYBR Green PCR Master mix, $2 \mu 1$ miScript Universal Primer, $0.5 \mu \mathrm{l}$ specific microRNA primer and $6.5 \mu \mathrm{l}$ RNase-free water. The sequence of the miR-510-5p forward primer was 5'-TAGCAGCACGTAAATATTGGCG-3' and the reverse primer was provided by the miScript SYBR Green PCR kit. The sequence of the U6 forward primer was 5'-CTCGCTTCGGCAGCACA-3' and reverse primer was 5'-ACGCTTCACGAATTTGCGT-3'. PCR amplification conditions were set as: $95^{\circ} \mathrm{C}$ for $2 \mathrm{~min}$, then 40 cycles of $95^{\circ} \mathrm{C}$ for $15 \mathrm{sec}, 58^{\circ} \mathrm{C}$ for $30 \mathrm{sec}$ and $72^{\circ} \mathrm{C}$ for $30 \mathrm{sec}$. The relative expression levels of miR-510-5p were calculated using the $2^{-\Delta \Delta \mathrm{Ct}}$ method (21).

Mature miRNA and negative control transfection. For the restoration of miR-510-5p, ACHN and 786-O cells were transfected with synthetic mature molecules (miR-510-5p mimics; Shanghai GenePharma Co., Ltd., Shanghai, China) with Lipofectamine 2000 (Invitrogen Life Technologies) according to the manufacturer's instructions. Mature miR-510-5p mimics and negative control were used in the gain-of-function experiments. The cancer cells were harvested and RNA was isolated for RT-qPCR to analyze the fold changes of miR-510-5p $24 \mathrm{~h}$ after transfection.

MTT assay. The capacity for cellular proliferation was determined using an MTT assay, according to the manufacturer's instructions. Approximately $5 \times 10^{3}$ cells were seeded into 96-well culture plates and transfected with 5 pmol miR-510-5p mimics or negative control. At 0, 24, 48 or $72 \mathrm{~h}$ after transfection, the cells were incubated with $20 \mu \mathrm{l}$ MTT $(5 \mu \mathrm{g} / \mathrm{ml})$ for $4 \mathrm{~h}$, followed by the addition of $150 \mu \mathrm{l}$ dimethyl sulfoxide (Sigma-Aldrich, St. Louis, MO, USA) and shaking for $15 \mathrm{~min}$ at room temperature to solubilize the crystals. The optical density (OD) was determined using a microplate reader (Model 680; Bio-Rad) at dual wavelength of 490/630 nm.

Flow cytometric analysis of apoptosis. Approximately 300,000 renal cancer cells were cultured in 6-well plates at $37^{\circ} \mathrm{C}$ and transfected with miR-510-5p mimics or negative control within $24 \mathrm{~h}$. Cancer cells, including floating cells, were harvested $48 \mathrm{~h}$ after transfection, washed twice with cold phosphate-buffered saline and resuspended in $100 \mu 1$ of $1 \mathrm{X}$ binding buffer (Invitrogen Life Technologies), followed by the addition of $5 \mu \mathrm{l}$ Annexin V-fluorescein isothiocyanate (Invitrogen Life Technologies) and $3 \mu$ propidium iodide (PI). The fluorescence of stained cells was then analyzed by flow cytometry (Beckman Coulter, Miami, FL, USA) using $488 \mathrm{~nm}$ excitation within $30 \mathrm{~min}$ of staining, according to the manufacturer's instructions.

Migration scratch assay. Wound scratch assay was used to assess the migration ability of 786-O and ACHN renal cancer cells in vitro. Approximately 350,000 cells were seeded per 12-well dish and transfected with 80 pmol miR-510-5p mimics or 80 pmol negative control $24 \mathrm{~h}$ later using Lipofectamine 2000.

After $5 \mathrm{~h}$ of transfection, the cell monolayer was scraped using a P-20 micropipette tip. The initial gap length $(0 \mathrm{~h})$ and the residual gap length $24 \mathrm{~h}$ after wound-healing were calculated using the software program MIAS-2000 (Leica Microsystems GmbH, Wetzlar, Germany). The experiments were performed in triplicate, repeated at least three times, and analyzed in a double-blind manner by at least two observers.

Bioinformatics. The potential targets of miR-510-5p were predicted by combining four public algorithms, miRanda (http://www.targetscan.org/), TargetScan (http://www. targetscan.org/), PicTar (http://pictar.mdc-berlin.de/) and miRWalk (http://www.umm.uni-heidelberg.de/apps/zmf/ mirwalk/). Putative genes predicted by all four algorithms were accepted and candidates were selected based on the gene function.

Statistical analysis. All statistical analysis was conducted with SPSS 17.0 statistical software package (SPSS Inc., Chicago, IL, USA). $\mathrm{P}<0.05$ was considered to indicate a statistically significant difference. The different expression of miR-510-5p in RCC and paired normal samples was analyzed by a paired t-test.

\section{Results}

miR-510-5p is downregulated in RCC tissues quantified by $R T$ - $q P C R$. Previous miRNA expression profiles of RCC indicated that miR-510-5p was downregulated $(22,23)$. In order 
Table I. Clinical and pathological features of 48 patients.

\begin{tabular}{lc}
\hline Variable & Number of cases \\
\hline Total & 48 \\
Age (years) & \\
$\geq 52$ & 29 \\
$<52$ & 19 \\
Gender & \\
Male & 30 \\
Female & 18 \\
Histological type & \\
Clear cell & 39 \\
Papillary & 9 \\
Primary tumor stage & \\
T1 & 27 \\
T2 & 19 \\
T3 and T4 & 2 \\
AJCC clinical stage & \\
I & 27 \\
II & 18 \\
III+IV & 3 \\
\hline &
\end{tabular}

AJCC, American Joint Committee on Cancer.

to confirm the results of former studies, RT-qPCR was used to quantify the expression of miR-510-5p in RCC and paired adjacent normal tissues from 48 patients. The results showed the expression of miR-510-5p decreased in $81.25 \%$ (39/48) of RCC tissues, compared with paired normal tissues, with an average reduction in expression of 0.4283 -fold (Fig. 1A). To investigate the effects of miR-510-5p on renal cancer cells, synthetic miR-510-5p mature mimics and negative controls were transfected into ACHN and 786-O cell lines. As shown in Fig.1B, RT-qPCR analysis indicated the fold changes of miR-510-5p in ACHN and 786-O cells after transfection were 9.58 and 11.32 , respectively.

Overexpression of miR-510-5p inhibits RCC cell proliferation. The impact on cell proliferation was analyzed by an MTT assay, OD values of two groups (miR-510-5p mimics and negative control) were measured at $0,24,48$ and $72 \mathrm{~h}$ after transfection. The present results showed the proliferation of ACHN cells decreased by $5.16 \%(\mathrm{P}>0.05), 15.26 \%(\mathrm{P}<0.05)$ and $29.06 \%(\mathrm{P}<0.05)$ while the proliferation of $786-\mathrm{O}$ cells decreased by $5.06(\mathrm{P}>0.05), 12.42(\mathrm{P}<0.05)$ and $21.78 \%$ $(\mathrm{P}<0.05)$ (Fig. 2), suggesting that miR-510-5p can reduce the proliferation of cancer cells.

Restoration of miR-510-5p induces RCC cell apoptosis. To demonstrate the effect of miR-510-5p on cell apoptosis, a flow cytometry assay was performed to detect the apoptosis rates of $\mathrm{ACHN}$ and 786-O cells after transfection. The results revealed that apoptosis rates of ACHN transfected with miR-510-5p mimics and negative control were 13.7 versus 5.0 while the apoptosis rates of 786-O cells were 10.8 versus $6.1(\mathrm{P}<0.05)$,
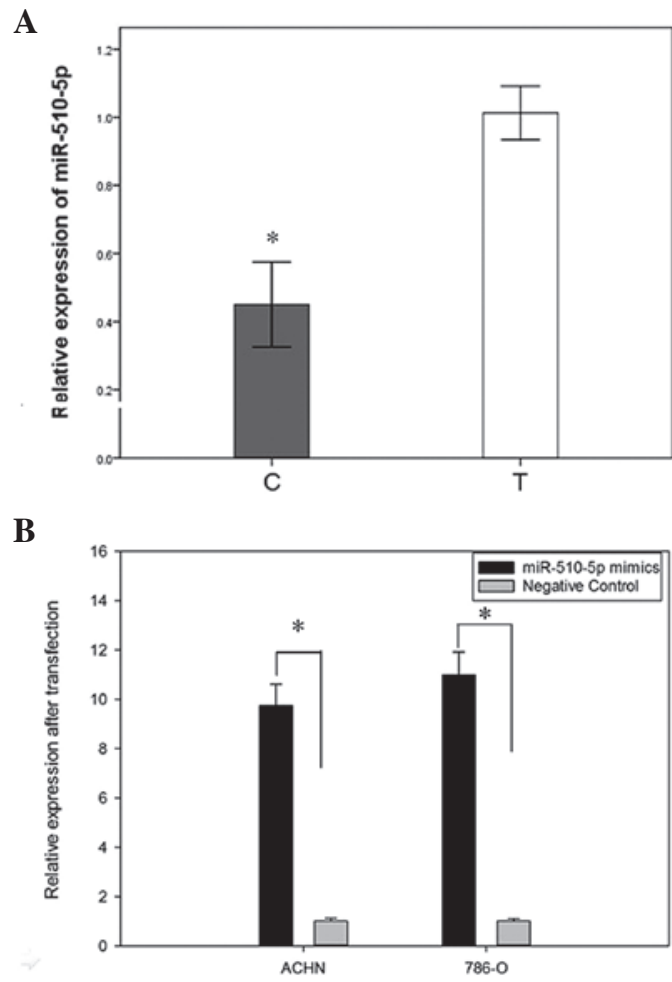

Figure 1. (A) Relative expression of miR-125a-5p in renal cell carcinoma (C) and normal tissues $(\mathrm{N})$ by reverse transcription-quantitative polymerase chain reaction. (B) Relative expression of miR-510-5p in ACHN and 786-O cells after transfection with miR-510-5p mimics. ${ }^{*} \mathrm{P}<0.05$ vs. negative control.

$\mathbf{A}$

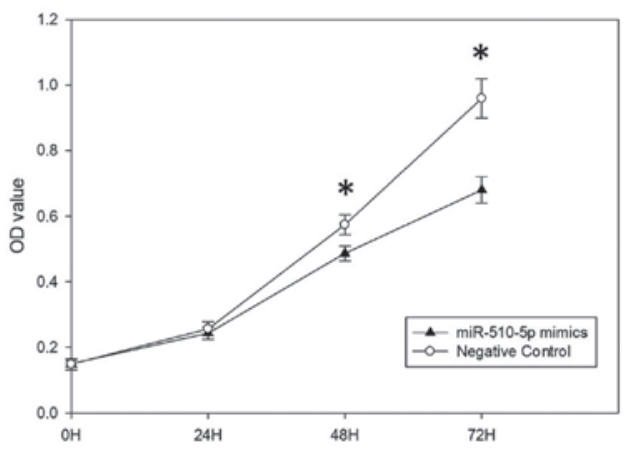

$\mathbf{B}$

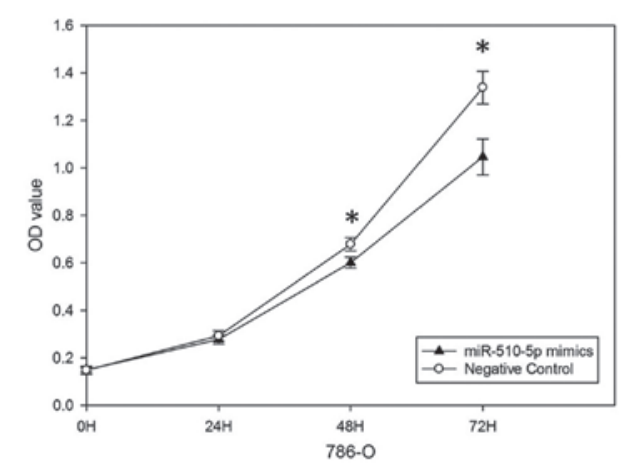

Figure 2. MTT assay for cell proliferation of ACHN and 786-O cells transfected with miR-510-5p mimics vs. negative control. (A) Cell proliferation of ACHN cells. (B) Cell proliferation of $786-\mathrm{O}$ cells. ${ }^{*} \mathrm{P}<0.05$, vs. negative control.

suggesting that restoration of miR-510-5p induces apoptosis of renal cancer cells (Fig. 3). 
Aa

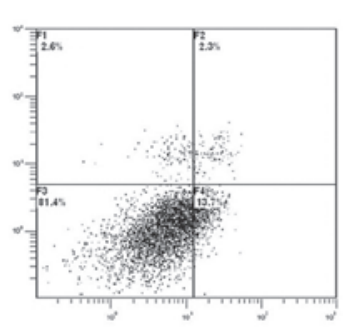

Ab

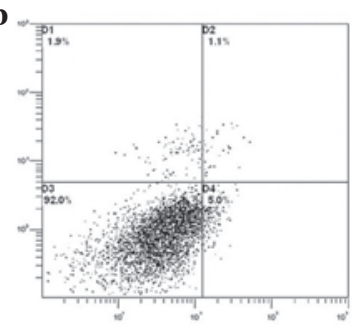

Ba

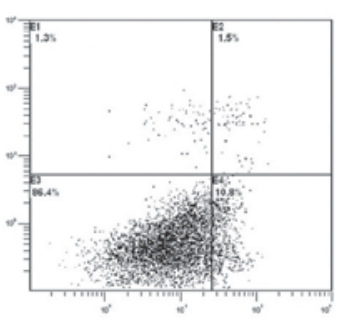

$\mathbf{B b}$

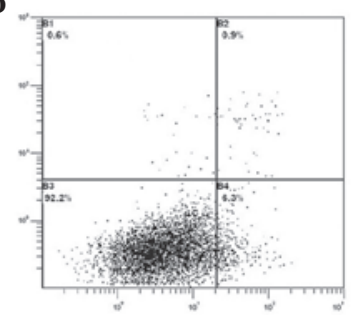

C

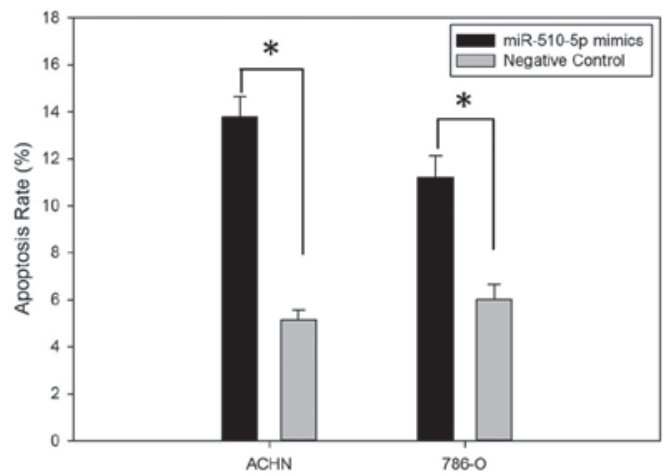

Figure 3. Flow cytometry to observe cell apoptosis of ACHN and 786-O cells after transfection with miR-510-5p mimics or negative control. (A) ACHN cells transfected with (Aa) miR-510-5p mimics and (Ab) negative control. (B) 786-O cells transfected with miR-510-5p mimics (Ba) and negative control (Bb) (C) Comparison of apoptosis rates of $\mathrm{ACHN}$ and $786-\mathrm{O}$ cancer cells transfected with miR-510-5p mimics and negative controls. ${ }^{* *} \mathrm{P}<0.01$ vs. negative control.

Aa

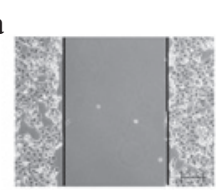

Ac

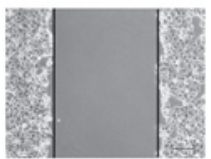

C

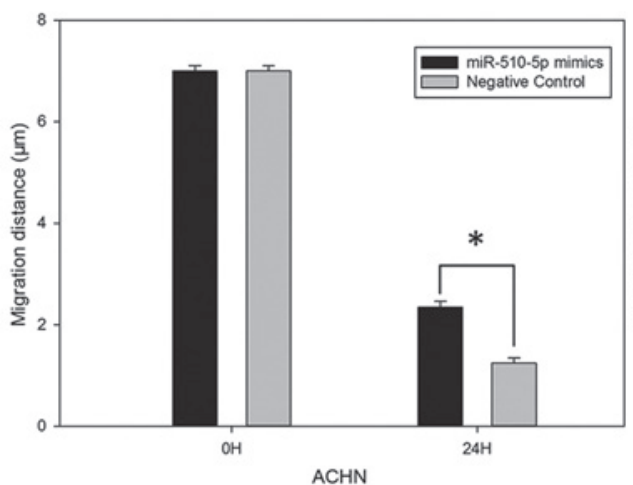

Ba

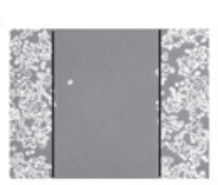

Bc

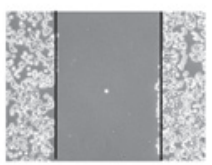

Bb

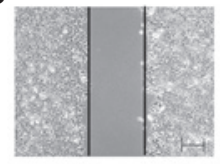

Bd

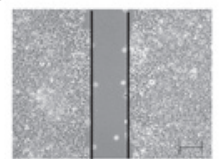

D

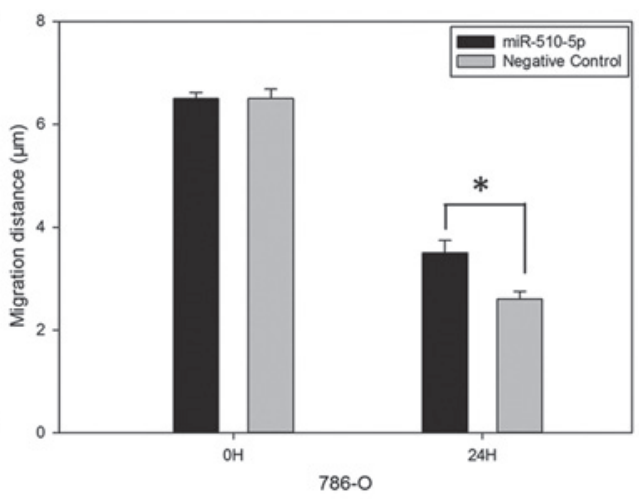

Figure 4. Wound scratch assay for ACHN and 786-O cells $24 \mathrm{~h}$ after transfection. (A) Images of the ACHN cells. Cells were transfected with negative control (Aa) $0 \mathrm{~h}$ and (Ab) $24 \mathrm{~h}$ after the wounds were made or with miR-510-5p mimics (Ac), $0 \mathrm{~h}$ and (Ad) $24 \mathrm{~h}$. (B)Images of the 786-O cells. Cells were transfected with negative control $(\mathrm{Ba}) 0 \mathrm{~h}$ and $(\mathrm{Bb}) 24 \mathrm{~h}$ after the wounds were made, or with miR-510-5p mimics at $(\mathrm{Bc}) 0 \mathrm{~h}$ and $(\mathrm{Bd}) 24 \mathrm{~h}$. Comparison of wound widths $(\mu \mathrm{m})$ in (C) ACHN cells and (D) 786-O cells using a standard caliper.

miR-510-5p mimics inhibited cell migration. The influence of miR-510-5p on cell migration was observed by a wound scratch assay. As presented in Fig. 4, wound width of the group transfected with miR-510-5p mimics was greater than that of the negative control group $(\mathrm{P}<0.05)$, suggesting that overex- pressed miR-510-5p inhibited the ability of migration of renal cancer cells (Fig. 4).

Target gene prediction. To investigate the downstream target genes of miR-510-5p, miRanda, TargetScan, PicTar and 


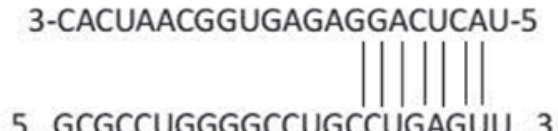

5...GCGCCUGGGGCCUGCCUGAGUU...3

3-CACUAACGGUGAGAGGACUCAU-5

5...GAAGAAAAUUGAGACCUGAGUG...3

3-CACUAACGGUGAGAGGACUCAU-5<smiles>C=C1C=CC=C1</smiles>

5...ACAACAAAUUCAG UUCUGAGUA...3
miR-510-5p

AKT2 3'UTR (310-321)

miR-510-5p

AKT3 3'UTR (1367-1388)

miR-510-5p

FAS 3'UTR (9-30)

Figure 5. Bioinformatics prediction of the target genes of miR-510-5p with complementary sites.

miRWalk were used in combination to predict the putative targets. AKT2, AKT3 and FAS were three of the potential targets predicted by all four algorithms simultaneously, of which the 3' untranslated region (UTR) of the mRNA contained a complementary site for the seed sequences of miR-510-5p (Fig. 5).

\section{Discussion}

Carcinogenesis is a complicated process that involves numerous genetic aberrations and signaling pathways. The recent identification of miRNAs and their capability of simultaneously regulating multiple downstream genes may be important in explaining the complex mechanisms underlying cancer formation (24). These short RNAs of 19-25 nucleotides are key in a wide variety of biological processes, including cell fate specification, proliferation, migration, apoptosis and tumorigenesis $(25,26)$. A number of studies have validated that miRNAs contribute to the development of various types of malignances, as well as to their invasive and metastatic capacities, including RCC (27). For example, miR-204 was confirmed to be a Von hippel-Lindau-regulated tumor suppressor acting by inhibiting macroautophagy, with MAP1LC3B (LC3B) as a direct and functional target (28). In addition, miR-21 regulates PTEN to force the canonical oncogenic Akt/TORC1 signaling conduit to drive renal cancer cell proliferation and invasion (29). Further research into the function and interaction with the target genes of deregulated miRNAs may reveal the molecular mechanisms underlying the tumorigenesis of RCC.

Aberrant expression of miR-510 has been observed in several types of cancer, as described above. In breast cancer, miR-510 directly binds to the 3'UTR of peroxiredoxin 1 and prevents its protein expression, thereby suppressing the migration of cancer cells (17). While in ovarian serous carcinoma (OSC), low miR-510 expression was significantly associated with poorer overall survival, indicating that miR-510 may be considered a novel-candidate clinical biomarker for predicting OSC outcome (18). However, the expression and function of miR-510 in RCC remains unclear.

In the present study, the expression of miR-510-5p in 48 paired RCC and normal renal specimens was quantified by
RT-qPCR and found that miR-510-5p was downregulated in RCC. The present results of decreased expression of miR-510-5p was in accordance with the results of recent miRNA expression profiles of RCC $(30,31)$. To investigate whether miR-510-5p was important for the tumorigenesis of RCC, MTT and wound scratch assays, as well as flow cytometry were used to analyze the impact of miR-510-5p on renal cancer by transfecting synthetic miR-510-5p mimics. The results show that cancer cells transfected with miR-510-5p mimics displayed less cellular proliferation and migration and more cellular apoptosis compared with the negative control groups, indicating that miR-510-5p may act as a tumor suppressor by reducing cell proliferation and migration, and inducing cell apoptosis in RCC.

It is generally acknowledged that miRNAs are important in various biological processes by 'imperfect' complementary binding to the 3'UTR of the downstream genes. To determine the target genes of miR-510-5p, several algorithms were combined to predict putative target genes and AKT2, AKT3 and FAS were identified as potential targets of miR-510-5p. AKT is a major transducer of the phosphoinositide 3-kinase (PI3K) pathway and is crucial in the regulation of cellular processes, such as growth, metabolism and survival. Mammalian cells are characterized by the expression of three different AKT isoforms (AKT1, AKT2 and AKT3), encoded by distinct genes (32). Emerging evidence has shown that AKT2 and AKT3 serve as significant contributors to malignancy (33). While FAS is a member of the TNF-receptor superfamily, which has been shown to be central in the physiological regulation of programmed cell death, and has been implicated in the pathogenesis of various malignancies (34). It has been reported that FAS expression is a surrogate biomarker of active cancer cell proliferation and accurately predicts RCC patient survival (35). Decreased expression of miR-510-5p may regulate cellular proliferation, migration and apoptosis by targeting oncogenes AKT2, AKT3 and FAS; however, this requires further research.

In conclusion, the present study revealed that downregulated miR-510-5p functioned as a tumor suppressor by reducing cellular proliferation and migration and inducing apoptosis in RCC. Further research is required to define target genes of miR-510-5p to elucidate the cellular mechanism underlying the effect of miR-510-5p in the carcinogenesis of RCC. 


\section{Acknowledgements}

This study was supported by the National Natural Science Foundation of China (no. 81101922), the Medical Scientific Research Foundation of Guangdong Province of China (nos. A2012584 and A2013606), the Science and Technology Development Fund Project of Shenzhen (grant no. JCYJ20130402114702124) and the fund of Guandong Key medical subject.

\section{References}

1. Cohen HT and McGovern FJ: Renal-cell carcinoma. N Engl J Med 353: 2477-2490, 2005.

2. Hofmann HS, Neef H, Krohe K, Andreev P and Silber RE: Prognostic factors and survival after pulmonary resection of metastatic renal cell carcinoma. Eur Urol 48: 77-81, 2005.

3. Arsanious A, Bjarnason GA and Yousef GM: From bench to bedside: current and future applications of molecular profiling in renal cell carcinoma. Mol Cancer 8: 20, 2009.

4. Wood CG: Molecular markers of prognosis in renal cell carcinoma: Insight into tumor biology helps define risk and provides targets for therapy. J Surg Oncol 94: 264-265, 2006.

5. Motzer RJ, Hutson TE, Tomczak P, et al: Sunitinib versus interferon alfa in metastatic renal-cell carcinoma. $\mathrm{N}$ Engl J Med 356: 115-124, 2007.

6. Patel C, Ahmed A and Ellsworth P: Renal cell carcinoma: a reappraisal. Urol Nurs 32: 182-190, 2012.

7. Maroto P and Rini B: Molecular biomarkers in advanced renal cell carcinoma. Clin Cancer Res 20: 2060-2071, 2014

8. Aqeilan RI, Calin GA and Croce CM: miR-15a and miR-16-1 in cancer: discovery, function and future perspectives. Cell Death Differ 17: 215-220, 2010.

9. White NM, Khella HW, Grigull J, et al: miRNA profiling in metastatic renal cell carcinoma reveals a tumour-suppressor effect for miR-215. Br J Cancer 105: 1741-1749, 2011.

10. Cho WC: OncomiRs: the discovery and progress of microRNAs in cancers. Mol Cancer 6: 60, 2007.

11. Tiwari M: Microarrays and cancer diagnosis. J Cancer Res Ther 8: 3-10, 2012.

12. Perez-Diez A, Morgun A and Shulzhenko N: Microarrays for cancer diagnosis and classification. Adv Exp Med Biol 593: 74-85, 2007.

13. Shenouda SK and Alahari SK: MicroRNA function in cancer: oncogene or a tumor suppressor? Cancer Metastasis Rev 28 369-378, 2009

14. Koberle V, Kronenberger B, Pleli T, et al: Serum microRNA-1 and microRNA-122 are prognostic markers in patients with hepatocellular carcinoma. Eur J Cancer 49: 3442-3449, 2013.

15. Zhao A, Li G, Péoc'h M, Genin C and Gigante M: Serum miR-210 as a novel biomarker for molecular diagnosis of clear cell renal cell carcinoma. Exp Mol Pathol 94: 115-120, 2013.

16. Patnaik SK, Kannisto E, Knudsen S and Yendamuri S: Evaluation of microRNA expression profiles that may predict recurrence of localized stage I non-small cell lung cancer after surgical resection. Cancer Res 70: 36-45, 2010.

17. Guo QJ, Mills JN, Bandurraga SG, et al: MicroRNA-510 promotes cell and tumor growth by targeting peroxiredoxin 1 in breast cancer. Breast Cancer Res 15: R70, 2013.
18. Yu X, Zhang X, Bi T, et al: MiRNA expression signature for potentially predicting the prognosis of ovarian serous carcinoma. Tumour Biol 34: 3501-3508, 2013.

19. Chen W, Tang Z, Sun Y, et al: miRNA expression profile in primary gastric cancers and paired lymph node metastases indicates that miR-10a plays a role in metastasis from primary gastric cancer to lymph nodes. Exp Ther Med 3: 351-356, 2012.

20. Guinan P, Sobin LH, Algaba F, et al: TNM staging of renal cell carcinoma: Workgroup No. 3. Union International Contre le Cancer (UICC) and the American Joint Committee on Cancer (AJCC). Cancer 80: 992-993, 1997

21. Schmittgen TD and Livak KJ: Analyzing real-time PCR data by the comparative C(T) method. Nat Protoc 3: 1101-1108, 2008.

22. White NM, Bao TT, Grigull J, et al: miRNA profiling for clear cell renal cell carcinoma: biomarker discovery and identification of potential controls and consequences of miRNA dysregulation. J Urol 186: 1077-1083, 2011.

23. Liu H, Brannon AR, Reddy AR, et al: Identifying mRNA targets of microRNA dysregulated in cancer: with application to clear cell renal cell carcinoma. BMC Syst Biol 4: 51, 2010.

24. Sana J, Faltejskova P, Svoboda M and Slaby O: Novel classes of non-coding RNAs and cancer. J Transl Med 10: 103, 2012.

25. Koscianska E, Baev V, Skreka K, et al: Prediction and preliminary validation of oncogene regulation by miRNAs. BMC Mol Biol 8: 79, 2007.

26. Volinia S, Galasso M, Costinean S, et al: Reprogramming of miRNA networks in cancer and leukemia. Genome Res 20: 589-599, 2010.

27. Kefas B, Godlewski J, Comeau L, et al: microRNA-7 inhibits the epidermal growth factor receptor and the Akt pathway and is down-regulated in glioblastoma. Cancer Res 68: 3566-3572, 2008.

28. Mikhaylova O, Stratton Y, Hall D, et al: VHL-regulated MiR-204 suppresses tumor growth through inhibition of LC3B-mediated autophagy in renal clear cell carcinoma. Cancer Cell 21: 532-546, 2012.

29. Dey N, Das F, Ghosh-Choudhury N, et al: microRNA-21 governs TORC1 activation in renal cancer cell proliferation and invasion. PLoS One 7: e37366, 2012

30. White NM, Bao TT, Grigull J, et al: miRNA profiling for clear cell renal cell carcinoma: Biomarker discovery and identification of potential controls and consequences of miRNA dysregulation. J Urol 186: 1077-1083, 2011.

31. Liu H, Brannon AR, Reddy AR, et al: Identifying mRNA targets of microRNA dysregulated in cancer: With application to clear cell renal cell carcinoma. BMC Syst Biol 4: 51, 2010.

32. Krzeslak A: Akt kinase: a key regulator of metabolism and progression of tumors. Postepy Hig Med Dosw (Online) 64: 490-503, 2010 (In Polish).

33. Agarwal E, Brattain MG and Chowdhury S: Cell survival and metastasis regulation by Akt signaling in colorectal cancer. Cell Signal 25: 1711-1719, 2013.

34. Macher-Goeppinger S, Bermejo JL, Wagener N, et al: Expression and prognostic relevance of the death receptor CD95 (Fas/APO1) in renal cell carcinomas. Cancer Lett 301: 203-211, 2011.

35. Sejima T, Morizane S, Hinata N, et al: Fas expression in renal cell carcinoma accurately predicts patient survival after radical nephrectomy. Urol Int 88: 263-270, 2012. 